\title{
Effects of spironolactone and losartan on the early neovascularization of acute myocardial infarction
}

\author{
YAN LIU ${ }^{1}$ and KUNSHEN LIU ${ }^{2}$ \\ ${ }^{1}$ Department of Geriatrics, The First Hospital of Shijiazhuang City, Shijiazhuang, Hebei 050011; ${ }^{2}$ Department of Cardiology, \\ The First Affiliated Hospital of Hebei Medical University, Shijiazhuang, Hebei 050031, P.R. China
}

Received March 28, 2014; Accepted May 16, 2014

DOI: $10.3892 /$ etm.2014.1791

\begin{abstract}
The aim of the present study was to investigate the effects of spironolactone and losartan on the early healing stage of acute myocardial infarction (AMI) in rats. An AMI rat model was established and the rats were randomly divided into four groups: AMI ( $\mathrm{n}=12), \mathrm{AMI}+$ spironolactone $(\mathrm{AMI}+\mathrm{S} ; \mathrm{n}=12), \mathrm{AMI}+\operatorname{losartan}(\mathrm{AMI}+\mathrm{L} ; \mathrm{n}=12)$ and AMI + spironolactone combined with losartan (AMI + S + L; $\mathrm{n}=12)$. Sham-operated rats served as a control group $(\mathrm{n}=12)$. The expression levels of matrix metalloproteinases (MMPs) and tissue inhibitors of matrix metalloproteinases (TIMPs) in the non-infarcted myocardium surrounding the AMI area were determined using immunohistochemistry. In addition, the capillary density in the non-infarcted myocardium surrounding the AMI area was detected. The capillary densities around the infarcted area in the AMI and treatment groups at day 7 and 14 following AMI surgery were significantly higher compared with the sham-operated rats. Compared with the AMI group, the capillary densities around the infarcted area and the ratio of MMPs/TIMP-1 were increased in the treatment groups following AMI surgery; however, the increased ratio of MMPs/TIMP-1 was reduced at day 14 following AMI surgery. Therefore, these results indicated that spironolactone and losartan may promote the formation of collateral circulation in the non-infarcted tissue surrounding the infarcted area by regulating the production of MMPs.
\end{abstract}

\section{Introduction}

Numerous studies have demonstrated that aldosterone is an important etiology of myocardial fibrosis and cardiac remodeling following myocardial infarction (1-4). Clinical trials conducted by the Randomized Aldactone Evaluation Study (5)

Correspondence to: Dr Yan Liu, Department of Geriatrics, The First Hospital of Shijiazhuang City, 36 Fanxi Road, Shijiazhuang, Hebei 050011, P.R. China

E-mail: yanliudoc@163.com

Key words: acute myocardial infarction, spironolactone, losartan, capillary density, matrix metalloproteinase and Eplerenone Post-Acute Myocardial Infarction Heart Failure Efficacy and Survival Study (6) have demonstrated that the receptor antagonists of aldosterone may significantly reduce cardiovascular-associated mortality in patients with severe heart failure. Recent studies have also shown that the receptor antagonists of mineralocorticoids may improve the survival of patients with systolic heart failure or mild to severe symptoms of heart failure (7-9). Clinical data have indicated that angiotensin-converting enzyme inhibitors are an effective therapeutic strategy for the treatment of heart failure to inhibit aldosterone and improve cardiac remodeling. However, due to their side effects, including an increase in the incidence of cough of $\leq 26 \%$, these drugs are not suitable for certain patients. Angiotensin receptor blockers selectively inhibit angiotensin 1 (AT1) and completely inhibit angiotensin II (Ang II). They exhibit similar efficacy and cardiac- and vascular-protective effects as angiotensin-converting enzyme inhibitors $(10,11)$. Therefore, these drugs may be used as an alternative for angiotensin-converting enzyme inhibitors in the treatment of heart failure.

Left ventricular remodeling is an important aim in the treatment of acute myocardial infarction (AMI) and heart failure (12). The degree of left ventricular remodeling following AMI is closely associated with severe left ventricular dysfunction and progression to congestive heart failure. The structure and function of the extracellular matrix (ECM) is maintained by matrix metalloproteinases (MMPs); however, degradation of the ECM is regulated by the endogenous tissue inhibitors of matrix metalloproteinases (TIMPs). Excessive degradation of components of the ECM leads to the expansion of the left ventricle and heart failure. Since TIMP-1 has a high affinity to a number of MMPs, binding to MMPs can inhibit the proteolytic activity of MMPs and have a direct role in left ventricular remodeling, indicating that TIMPs may be used to predict the clinical outcomes of patients with heart failure $(13,14)$. TIMPs form MMP-TIMP complexes with MMPs in a 1:1 ratio to inhibit the binding of MMPs to their substrates. If the ratio of MMPs/TIMP-1 increases, the degradation of the ECM is accelerated. MMP-2 and MMP-9 are two gelatinases that degrade type IV and type V collagens. However, the degradation of type IV and V collagens is an indication of inflammation-mediated angiogenesis.

Michel et al (15) demonstrated that aldosterone may be used to induce the formation of new blood vessels in ischemic 
tissues via the Ang II pathway, which was not completely inhibited by $20 \mathrm{mg} / \mathrm{day} / \mathrm{kg}$ spironolactone, a receptor antagonist of aldosterone, or $20 \mathrm{mg} /$ day/ $\mathrm{kg}$ valsartan, a type I receptor antagonist of Ang II. In the present study, the effect of higher doses of aldosterone receptor antagonist and AT1 receptor antagonist on the angiogenesis in the surrounding tissues of an AMI area was investigated. An AMI rat model was treated with a large dose of spironolactone $(80 \mathrm{mg} / \mathrm{day} / \mathrm{kg})$ and/or losartan $(10 \mathrm{mg} / \mathrm{day} / \mathrm{kg})$ to investigate the changes in the expression levels of MMP-2, MMP-9 and TIMP-1, as well as the capillary density, in the non-infarcted area surrounding the infarcted area. The aim of the study was to observe the effects of spironolactone and/or losartan on the early neovascularization in AMI rats.

\section{Materials and methods}

Animals. A total of 60 healthy male Wister rats, weighing between 220 and $250 \mathrm{~g}$, were obtained from the Experimental Animal Center of Hebei Medical University (Shijiazhuang, China). The rats were randomly divided into sham-operated $(n=12)$ and AMI groups $(n=48)$. The animals used in the study were handled and treated in accordance with the strict guiding principles of the National Institutes of Health for the Experimental Care and Use of Animals. The experimental design and procedures were approved by the Institutional Ethical Committee for Animal Care and Use of Hebei Medical University.

Establishment of the AMI rat model and treatment. In the AMI group, the left anterior descending coronary artery of the rats was ligated to induce a large area of AMI. Sham-operated rats underwent the same surgery, but without ligation. Following surgery, all the animals were housed alone at a constant temperature of $37^{\circ} \mathrm{C}$. After $72 \mathrm{~h}$, the surviving rats in the AMI group were randomly divided into four groups and treated as follows. The AMI group $(n=12)$ were treated with physiological saline. The AMI + spironolactone group (AMI + S group, $\mathrm{n}=12$ ) were administered $80 \mathrm{mg} / \mathrm{day} / \mathrm{kg}$ spironolactone intragastrically, while the AMI + spironolactone + losartan group (AMI $+\mathrm{S}+\mathrm{L}$ group, $\mathrm{n}=12$ ) were administered $80 \mathrm{mg} /$ day/kg spironolactone and $10 \mathrm{mg} /$ day/ $\mathrm{kg}$ losartan intragastrically. The AMI + losartan group (AMI + L group, $n=12)$ were administered $10 \mathrm{mg} / \mathrm{day} / \mathrm{kg}$ losartan intragastrically and the sham-operated group were administered an equal volume of physiological saline.

Sampling. At day 7 and 14 following AMI surgery, six rats in each group were anesthetized with $10 \%$ chloral hydrate, and the heart was quickly removed. The hearts were rapidly placed into $4 \%$ paraformaldehyde solution and fixed for $12-24 \mathrm{~h}$. Following dehydration using gradient concentration $(70 \%$, $80 \%, 95 \%$ and $100 \%$ ) of alcohol-water solution, the heart tissues were embedded in paraffin blocks. Serial sections of $5 \mu \mathrm{m}$-thickness were mounted on poly-L-lysine-coated slides and used for hematoxylin and eosin (HE) and immunohistochemical staining of TIMP-1, MMP-2 and MMP-9.

Immunohistochemical analysis [Streptavidin peroxidase (SP) method]. Heart tissue sections were deparaffinized to hydro- philia and incubated with $3 \% \mathrm{H}_{2} \mathrm{O}_{2}$ in methanol for 10 min at room temperature to remove the endogenous peroxidase activity. The sections were rinsed with distilled water and 0.01 M phosphate-buffered saline (PBS; $\mathrm{pH} 7.4$ ). Slides were microwaved in $0.1 \mathrm{M}$ citrate buffer ( $\mathrm{pH}$ 6.0) at $98^{\circ} \mathrm{C}$ for $20 \mathrm{~min}$ and then cooled to room temperature, which was followed by incubation with $10 \%$ normal rabbit serum in $0.1 \mathrm{M}$ PBS ( $\mathrm{pH}$ 7.4) to block the non-specific sites at room temperature for $30 \mathrm{~min}$. Respective incubation with rabbit anti-rat TIMP-1, goat anti-rat MMP2 and MMP9 (all diluted 1:50 in PBS; Beijing ZSGB-Biotechnology, Co., Ltd., Beijing, China) was performed overnight at $4^{\circ} \mathrm{C}$ in a humidified chamber, prior to incubation with secondary biotin-conjugated antibodies and sequential incubation with horseradish peroxidase-conjugated streptavidin and the peroxidase substrate, 3'-diaminobenzidine (all from Beijing ZSGB-Biotechnology, Co., Ltd., Beijing, China). Samples were then counterstained with hematoxylin. PBS was used instead of a primary antibody as a negative control.

Evaluation of immunohistochemical staining. Positive immunoreactions for TIMP-1, MMP-2 and MMP-9 presented with brown or yellow staining in the cytoplasm. Under a microscope (Microphot-FXA, Nikon Corp., Shizuoka, Japan), 10 randomly selected fields in each slide were observed and 15 positive sites in each field were imaged in order to calculate the gray values using imaging analysis system software (Beihang University, Beijing, China). The mean gray values of each slide were calculated and compared.

Determination of capillary density. Slides with HE staining were observed under a light microscope. The number of capillaries in 10 randomly selected fields (magnification, x200) in each section were calculated. Results were expressed as the average of the numbers $/ \mathrm{mm}^{2}$.

Statistical analysis. Results are expressed as the mean \pm standard deviation. Normal distribution of the data was first verified. If normal, comparisons between the groups were performed using one-way analysis of variance. Comparisons between the sham-operated and AMI groups were conducted using the least significant difference test, while comparisons between any two groups were performed using the Student-Newman-Keuls test. All statistical analysis was performed using SPSS 10.0 software (SPSS, Inc., Chicago, IL, USA), and $\mathrm{P}<0.05$ was considered to indicate a statistically significant difference.

\section{Results}

Effect of spironolactone and/or losartan on angiogenesis following AMI. At day 7 and 14 following AMI surgery, the rats treated with spironolactone and/or losartan presented higher capillary density in the non-infarcted tissue around the infarcted area when compared with the AMI or sham-operated groups (Table I).

MMP-2 expression in the non-infarcted tissue around the infarcted area. At day 7 following AMI surgery, only the $\mathrm{AMI}+\mathrm{S}$ group exhibited higher expression levels of MMP-2 
Table I. Expression levels of MMP-2, MMP-9 and TIMP-1, and the capillary density of each group.

\begin{tabular}{|c|c|c|c|c|}
\hline Groups & MMP-2 & MMP-9 & TIMP-1 & $\begin{array}{l}\text { Capillary density, } \\
\text { capillaries } / \mathrm{mm}^{2}\end{array}$ \\
\hline \multicolumn{5}{|c|}{ Sham-operated } \\
\hline Day 7 & $0.166 \pm 0.015$ & $0.172 \pm 0.016$ & $0.231 \pm 0.014$ & $13.384 \pm 0.293$ \\
\hline Day 14 & $0.153 \pm 0.015$ & $0.171 \pm 0.016$ & $0.236 \pm 0.014$ & $13.426 \pm 0.226$ \\
\hline \multicolumn{5}{|l|}{ AMI } \\
\hline Day 7 & $0.184 \pm 0.013$ & $0.203 \pm 0.014$ & $0.228 \pm 0.014$ & $14.574 \pm 0.374$ \\
\hline Day 14 & $0.162 \pm 0.013$ & $0.191 \pm 0.015$ & $0.199 \pm 0.018$ & $14.482 \pm 0.262$ \\
\hline \multicolumn{5}{|l|}{$\mathrm{AMI}+\mathrm{S}$} \\
\hline Day 7 & $0.197 \pm 0.013$ & $0.192 \pm 0.015$ & $0.207 \pm 0.016$ & $15.754 \pm 0.464$ \\
\hline Day 14 & $0.146 \pm 0.012$ & $0.178 \pm 0.013$ & $0.236 \pm 0.016$ & $20.225 \pm 0.513$ \\
\hline \multicolumn{5}{|l|}{$\mathrm{AMI}+\mathrm{L}$} \\
\hline Day 7 & $0.176 \pm 0.012$ & $0.186 \pm 0.016$ & $0.204 \pm 0.018$ & $18.575 \pm 0.266$ \\
\hline Day 14 & $0.151 \pm 0.093$ & $0.172 \pm 0.085$ & $0.225 \pm 0.014$ & $19.483 \pm 0.403$ \\
\hline \multicolumn{5}{|c|}{$\mathrm{AMI}+\mathrm{S}+\mathrm{L}$} \\
\hline Day 7 & $0.182 \pm 0.088$ & $0.206 \pm 0.016$ & $0.197 \pm 0.014$ & $16.834 \pm 0.437$ \\
\hline Day 14 & $0.153 \pm 0.087$ & $0.174 \pm 0.013$ & $0.236 \pm 0.015$ & $20.455 \pm 0.405$ \\
\hline F-value & 58.72 & 60.58 & 34.51 & 110.84 \\
\hline P-value & $<0.01$ & $<0.01$ & $<0.01$ & $<0.01$ \\
\hline
\end{tabular}

Data are expressed as the mean \pm standard deviation. MMP, matrix metalloproteinase; TIMP, tissue inhibitor of matrix metalloproteinase; AMI, acute myocardial infarction; S, spironolactone; L, losartan. The F and P-values refer to the comparison result of day 14 following AMI surgery among multiple groups using one-way analysis of variance.

Table II. Ratios of MMP-2/TIMP-1 and MMP-9/TIMP-1 in each group.

\begin{tabular}{|c|c|c|c|c|c|}
\hline Parameters & Sham-operated & AMI & $\mathrm{AMI}+\mathrm{S}$ & $\mathrm{AMI}+\mathrm{L}$ & $\mathrm{AMI}+\mathrm{S}+\mathrm{L}$ \\
\hline \multicolumn{6}{|c|}{ MMP-2/TIMP-1 } \\
\hline Day 7 & 0.719 & 0.807 & 0.952 & 0.863 & 0.924 \\
\hline Day 14 & 0.648 & 0.814 & 0.619 & 0.671 & 0.648 \\
\hline \multicolumn{6}{|c|}{ MMP-2/TIMP-1 } \\
\hline Day 7 & 0.745 & 1.009 & 0.952 & 0.912 & 1.046 \\
\hline Day 14 & 0.725 & 0.962 & 0.754 & 0.764 & 0.737 \\
\hline
\end{tabular}

MMP, matrix metalloproteinase; TIMP, tissue inhibitor of matrix metalloproteinase; AMI, acute myocardial infarction; S, spironolactone; L, losartan.

in the non-infarcted tissue surrounding the infarcted area when compared with the AMI group $(\mathrm{P}<0.01)$. However, at day 14 following AMI surgery, the increased expression of MMP-2 was reduced significantly compared with the AMI group $(\mathrm{P}<0.01$; Table I).

MMP-9 expression in the non-infarcted tissue around the infarcted area. At day 14 following AMI surgery, the three treatment groups exhibited significantly lower expression levels of MMP-9 in the non-infarcted tissue around the infarcted area when compared with the AMI group $(\mathrm{P}<0.01)$, and similar levels to that of the sham-operated group (Table I).
Changes in the expression levels of TIMP-1 in the non-infarcted tissue around the infarcted area following treatment. TIMP-1 expression levels in the non-infarcted tissue around the infarcted area were markedly lower in the three treatment groups at day 7 following AMI surgery when compared with the AMI and sham-operated groups $(\mathrm{P}<0.01)$. However, at day 14 following AMI surgery, the expression increased gradually and the levels were higher than those in the AMI group $(\mathrm{P}<0.01)$ and close to the level in the sham-operated group (Table I).

MMPS/TIMP-1 ratio in the non-infarcted tissue around the infarcted area was significantly affected by spironolactone 
and/or losartan. Compared with the AMI group, the ratios of MMPs/TIMP-1 in the three treatment groups were increased at day 7 following AMI surgery; however, the ratio then increased to a normal level at day 14 following AMI surgery (Table II).

\section{Discussion}

Aldosterone has been previously demonstrated to significantly enhance ischemia-induced angiogenesis via the Ang II pathway (1) and increase the expression of vascular endothelial growth factor by 2.3 -fold. However, $20 \mathrm{mg} /$ day $/ \mathrm{kg}$ spironolactone or $20 \mathrm{mg} / \mathrm{day} / \mathrm{kg}$ valsartan does not completely inhibit this effect. Thus, the present study investigated the effects of higher doses of aldosterone receptor antagonist and AT1 receptor antagonist in the inhibition of vascularization in the surrounding area of the infarcted area. The effects of high doses of the aldosterone receptor antagonist, spironolactone (80 $\mathrm{mg} / \mathrm{day} / \mathrm{kg})$, and the AT1 receptor antagonist, losartan (10 mg/day/kg), were analyzed in the early stages of vascularization in AMI rats. The results demonstrated that high doses of spironolactone $(80 \mathrm{mg} / \mathrm{day} / \mathrm{kg})$ and losartan $(10 \mathrm{mg} / \mathrm{day} / \mathrm{kg})$ significantly increased the ratios of MMPs/TIMP-1 in the non-infarcted tissue surrounding the infarcted area in AMI rats at day 7 following AMI surgery. However, the ratios were reduced to a normal level at day 14 following AMI surgery. The application of spironolactone and losartan may also enhance the capillary density in the non-infarcted tissue surrounding the infarcted area in AMI rats, indicating that spironolactone and losartan have a promotive effect on angiogenesis in the non-infarcted tissue surrounding the infarcted area in AMI rats.

Selective degradation of the ECM to adjust the morphology, growth and survival of vascular endothelial cells can affect the growth and degradation of capillaries during angiogenesis (16), while the selective secretion and activation of MMPs indicates the changes in cell-cell junctions and the extension of cells. MMP-2 and MMP-9 are two gelatinases that hydrolyze collagen IV and collagen V. If collagen IV and V are degraded, the ECM molecules change, activating vascular endothelial cells via intracellular signaling pathways, which promotes the formation of neovessels. Johnson et al (17) demonstrated that the capillary density in the ischemic tissue of MMP-9-/- mice was not significantly different compared with the density prior to AMI surgery, while the capillary density in the ischemic tissue of wild-type mice increased by $39 \%$. These observations indicate that activation of MMP-9 is essential for a variety of physiological and pathological processes in the angiogenesis of ischemic tissues. Yan et al (18) found that reducing the cleavage of pro-MMP-2 increased fibronectin density in the cultured endothelial cells and caused cells to adhere and extend, indicating that MMP-2 may regulate vascular endothelial cells. A study by Haas et al (19) demonstrated that the hydrolysis of the basement membrane proteins of capillaries is one indication of inflammatory-mediated angiogenesis. TIMPs are specific endogenous inhibitors of MMPs. Dynamic alteration in the ratio of MMPs/TIMPs may result in the selective degradation of ECM, thereby affecting the growth and degradation of capillaries during angiogenesis.

The effects of spironolactone and/or losartan on neovascularization in the non-infarcted tissue surrounding the infarcted area were also investigated in the present study. The expression levels of MMP-2, MMP-9 and TIMP-1 were determined in the non-infarcted tissue surrounding the infarcted area of the AMI rats treated with or without spironolactone and/or losartan. In addition, the ratios of MMPs/TIMP-1 in the non-infarcted tissue surrounding the infarcted area of AMI rats were increased by spironolactone and/or losartan at day 7 following AMI surgery; however, the ratios then reduced to normal levels at day 14 following AMI surgery. These results indicate that the application of spironolactone and losartan in the early stages of AMI may promote angiogenesis in the non-infarcted tissue surrounding the infarcted area of AMI rats via dynamic changes between the levels of TIMP-1 and MMP-2 and MMP-9. The elevated ratios of MMP-2/TIMP-1 and MMP-9/TIMP-1 accelerated the degradation of non-fibrous collagen in the basement membrane, thereby inducing angiogenesis and promoting the formation of collateral circulation.

The production of aldosterone and Ang II in the adrenal glands and non-infarcted myocardium of AMI rats has been shown to be significantly increased. In addition, aldosterone has been demonstrated to significantly enhance the induction of ischemia on the formation of new blood vessels via the Ang II pathway. However, high doses of spironolactone $(80 \mathrm{mg} / \mathrm{day} / \mathrm{kg}$ ) did not inhibit the proinflammatory effects of aldosterone (20). Silvestre et al (20) found that the mRNA expression of aldosterone synthase and the levels of Ang II were significantly higher in the non-infarcted area of AMI rats that had been treated with $80 \mathrm{mg} / \mathrm{day} / \mathrm{kg}$ spironolactone at day 25 following AMI surgery. These observations may be the result of negative feedback or that aldosterone did not entirely exert its effects, including proinflammatory, promotion of tissue-repairing and enhancing ischemia-induced angiogenesis, via the aldosterone receptors or AT1 receptors. However, the exact mechanism remains to be elucidated.

Drüppel et al (21) demonstrated that aldosterone induced stiffed endothelial cell syndrome (SECS), characterized by the upregulation of epithelial sodium channels (ENaCs) and the mechanical enhancements of the endothelial cell cortex, combined with vascular endothelial dysfunction. In vivo, the antagonism of aldosterone has a continual protective role on the cardiovascular system. However, in cultured endothelial cells, the expression of the mineralocorticoid receptor was increased by $129 \%$. The expression of ENaCs increased by 32 and their surface area increased by $42 \%$ (from 13.8 to 19.6), respectively. However, the receptor was inhibited by spironolactone. Following three weeks of treatment, the release of nitric oxide was elevated by $50 \%$. Thus, spironolactone was shown to prevent the time-dependent performance of SECS by long-lasting improvement of endothelial function, which emphasizes the critical role of vascular endothelial cells as a therapeutic target in cardiovascular disease.

In conclusion, the results of the present study demonstrated that the application of high doses of aldosterone receptor antagonist and AT1 receptor antagonist during the early stages following AMI surgery is able to inhibit angiogenesis in the surrounding area. However, the optimal dose and time for the early application of spironolactone and the exact effects on cardiac function require further investigation, as well as the mechanisms underlying the function of aldosterone receptor antagonists in AMI. 


\section{References}

1. McKelvie RS, Moe GW, Cheung A, Costigan J, et al: The 2011 Canadian Cardiovascular Society heart failure management guidelines update: focus on sleep apnea, renal dysfunction, mechanical circulatory support, and palliative care. Can J Cardiol 27: 319-338, 2011.

2. Hayashi M, Tsutamoto T, Wada A, et al: Relationship between transcardiac extraction of aldosterone and left ventricular remodeling in patients with first acute myocardial infarction: extracting aldosterone through the heart promotes ventricular remodeling after acute myocardial infarction. J Am Coll Cardiol 38: 1375-1382, 2001

3. Leopold JA: Aldosterone, mineralocorticoid receptor activation, and cardiovascular remodeling. Circulation 124: e466-e468, 2011.

4. Sun Y, Zhang J, Lu L, Chen SS, Quinn MT and Weber KT: Aldosterone-induced inflammation in the rat heart: role of oxidative stress. Am J Pathol 161: 1773-1781, 2002.

5. Zannad F, Alla F, Dousset B, Perez A and Pitt B: Limitation of excessive extracellular matrix turnover may contribute to survival benefit of spironolactone therapy in patients with congestive heart failure: insights from the randomized aldactone evaluation study (RALES). Rales Investigators. Circulation 102: 2700-2706, 2000

6. Pitt B, Ruilope L, Bakris G, et al: EPHESUS Investigators: 459 Risk/benefit analysis of hyperkalemia in the eplerenone post-acute myocardial infarction heart failure efficacy and survival study (EPHESUS). Eur J Heart Fail 3 (Supp 1): 116 2004.

7. Zannad F, Stough WG, Rossignol P, et al: Mineralocorticoid receptor antagonists for heart failure with reduced ejection fraction: integrating evidence into clinical practice. Eur Heart J 33: 2782-2795, 2012.

8. Butler J, Ezekowitz JA, Collins SP, et al: Update on aldosterone antagonists use in heart failure with reduced left ventricular ejection fraction. Heart Failure Society of America Guidelines Committee. J Card Fail 18: 265-281, 2012.

9. Zannad F, McMurray JJ, Krum H, et al. Eplerenone in patients with systolic heart failure and mild symptoms. N Engl J Med 364: $11-21,2011$

10. Bhakta S and Dunlap ME: Angiotensin-receptor blockers in heart failure: evidence from the CHARM trial. Cleve Clin J Med 71: 665-673, 2004
11. Krum H, Carson P, Farsang C, et al: Effect of valsartan added to background ACE inhibitor therapy in patients with heart failure: results from Val-HeFT. Eur J Heart Fail 6: 937-945, 2004.

12. Mann DL and Bristow MR: Mechanisms and models in heart failure: the biomechanical model and beyond. Circulation 111: 2837-2849, 2005.

13. Stockand JD and Meszaros JG: Aldosterone stimulates proliferation of cardiac fibroblasts by activating Ki-RasA and MAPK1/2 signaling. Am J Physiol Heart Circ Physiol 284: H176-H184, 2003.

14. Chancey AL, Brower GL, Peterson JT and Janicki JS: Effects of matrix metalloproteinase inhibition on ventricular remodeling due to volume overload. Circulation 105: 1983-1988, 2002.

15. Michel F, Ambroisine ML, Duriez M, Delcayre C, Levy BI and Silvestre JS: Aldosterone enhances ischemia-induced neovascularization through angiotensin II-dependent pathway. Circulation 109: 1933-1937, 2004.

16. Davis GE, Pintar Allen KA, Salazar R and Maxwell SA: Matrix metalloproteinase- 1 and -9 activation by plasmin regulates a novel endothelial cell-mediated mechanism of collagen gel contraction and capillary tube regression in three-dimensional collagen matrices. J Cell Sci 114: 917-930, 2001.

17. Johnson C, Sung HJ, Lessner SM, Fini ME and Galis ZS: Matrix metalloproteinase- 9 is required for adequate angiogenic revascularization of ischemic tissues: potential role in capillary branching. Circ Res 94: 262-268, 2004.

18. Yan L, Moses MA, Huang S and Ingber DE: Adhesion-dependent control of matrix metalloproteinase-2 activation in human capillary endothelial cells. J Cell Sci 113: 3979-3987, 2000.

19. Haas TL, Milkiewicz M, Davis SJ, et al: Matrix metalloproteinase activity is required for activity-induced angiogenesis in rat skeletal muscle. Am J Physiol Heart Circ Physiol 279: H1540-H1547, 2000.

20. Silvestre JS, Heymes C, Oubénaïssa A, et al: Activation of cardiac aldosterone production in rat myocardial infarction: effect of angiotensin II receptor blockade and role in cardiac fibrosis. Circulation 99: 2694-2701, 1999.

21. Drüppel V, Kusche-Vihrog K, Grossmann C, et al: Long-term application of the aldosterone antagonist spironolactone prevents stiff endothelial cell syndrome. FASEB J 27: 3652-3659, 2013. 